Transactions of the Royal Society of Tropical Medicine and Hygiene. 2021 Jul 23;trab104.

doi: $10.1093 /$ trstmh/trab104.

\title{
Prevalence of Strongyloides stercoralis in the immunocompetent and immunocompromised individuals in Iran: a systematic review and meta- analysis
}

Aida Vafae Eslahi ${ }^{1}$, Meysam Olfatifar ${ }^{2}$, Elham Houshmand ${ }^{3}$, Morteza Ghanbari Johkool ${ }^{4}$, Mohammad Zibaei ${ }^{5}$, Masoud Foroutan ${ }^{6}$, Hamid Hosseini ${ }^{5}$, Milad Badri ${ }^{7}$

${ }^{1}$ Clinical Research Development Unit, Velayat Hospital, Qazvin University of Medical Sciences, Shahid Bahonar Boulevard, Qazvin, Iran.

${ }^{2}$ Gastroenterology and Liver Diseases Research Center, Research Institute for Gastroenterology and Liver Diseases, Shahid Beheshti University of Medical Sciences, Tehran Province, Tehran, Velenjak, 7th Floor, Bldg No.2 SBUMS, Arabi Ave, Tehran, Iran.

${ }^{3}$ Department of Parasitology, Faculty of Veterinary Medicine, Islamic Azad University, Rasht Branch, Guilan, Iran.

${ }^{4}$ Metabolic Diseases Research Center, Research Institute for Prevention of Non-Communicable Diseases, Qazvin University of Medical Sciences, Shahid Bahonar Boulevard, Qazvin, Iran.

${ }_{5}^{5}$ Department of Parasitology and Mycology, School of Medicine, Alborz University of Medical Sciences, Taleghani Boulevard, Taleghani square, Karaj, Iran.

${ }^{6}$ Department of Medical Parasitology, Faculty of Medicine, Abadan University of Medical Sciences, Zolfaghari 30m st, Abadan, Iran.

${ }^{7}$ Medical Microbiology Research Center, Qazvin University of Medical Sciences, Shahid Bahonar Boulevard, Qazvin, Iran.

\begin{abstract}
Strongyloidiasis is a neglected tropical disease mostly distributed in tropical and subtropical regions. The current study evaluated the prevalence of Strongyloides stercoralis in immunocompetent and immunodeficient patients in Iran. The available online literature published from June 1994 to October 2020 was obtained from multiple English databases (PubMed, Science Direct, Scopus, Web of Science and Google Scholar) and four Persian databases (Magiran, Iran Medex, Iran Doc and SID). All statistical analyses were performed using R software (version 3.6) meta-package and p-values $<0.05$ were considered significant. From 1051 articles, 74 studies (248 656 individuals) met the inclusion criteria. The pooled prevalence of S. stercoralis was $2 \%$ (95\% confidence interval [CI] 1 to 3) and 4\% (95\% CI 1 to 8) in immunocompetent and immunodeficient patients, respectively. In immunodeficient cases, the pooled prevalence of studies utilizing serology, culture and microscopic methods was 10\% (95\% CI 2 to 23), 1\% (95\% CI 0 to 6) and $1 \%$ (95\% CI 0 to 1), respectively. In immunocompetent cases, the pooled prevalence of studies utilizing microscopic, culture and molecular methods was 2\% (95\% CI 1 to 3), $2 \%$ (95\% CI 1 to 4) and 2\% (95\% CI 0 to 6), respectively. We propose an appropriate screening and control program along with comprehensive research regarding the frequency of strongyloidiasis in the country.
\end{abstract}

Keywords: Strongyloides stercoralis; Iran; neglected tropical disease; soil-transmitted helminth; systematic review. 